\title{
Transcriptional regulation of MdPIN3 and MdPIN10 by MdFLP during apple self- rooted stock adventitious root gravitropism
}

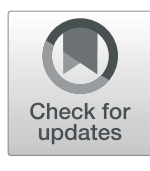

Zenghui Wang, Jialin Li, Yunfei Mao, Manman Zhang, Rong Wang, Yanli Hu, Zhiquan Mao and Xiang Shen*

\begin{abstract}
Background: The close planting of dwarfing self-rooted rootstocks is currently a widely used method for apple production; however, self-rooted rootstocks are weak with shallow roots and poor grounding. Therefore, understanding the molecular mechanisms that establish the gravitropic set-point angles (GSAs) of the adventitious roots of self-rooted apple stocks is important for developing self-rooted apple rootstock cultivars with deep roots.

Results: We report that the apple FOUR LIPS (MdFLP), an R2R3-MYB transcription factor (TF), functions in establishing the GSA of the adventitious roots of self-rooted apple stocks in response to gravity. Biochemical analyses demonstrate that MdFLP directly binds to the promoters of two auxin efflux carriers, MdPIN3 and MdPIN10, that are involved in auxin transport, activates their transcriptional expression, and thereby promotes the development of adventitious roots in self-rooted apple stocks. Additionally, the apple auxin response factor MdARF19 influences the expression of those auxin efflux carriers and the establishment of the GSA of adventitious roots of apple in response to gravity by directly activating the expression of MdFLP.
\end{abstract}

Conclusion: Our findings provide new insights into the transcriptional regulation of MdFLP by the auxin response factor MdARF19 in the regulation of the GSA of adventitious roots of self-rooted apple stocks in response to gravity.

Keywords: Gravitropic set-point angle, Auxin, Adventitious roots of self-rooted apple stocks, FOUR LIPS, Auxin response factor 19, PIN-FORMED

\section{Background}

The plant hormone auxin, which integrates many endogenous and environmental signals regulating lateral root formation [17], commonly plays a key role in embryogenesis, organogenesis, morphogenesis, and cell determination and division $[33,48]$. In the lateral root cap, the auxin precursor indole-3-butyric acid is converted to indole-3-acetic acid (IAA), and auxin is sent into the root clock [24], thus stimulating the formation of the prebranch site [45]. Subsequent lateral root patterning and morphogenesis are coordinated by dynamic auxin flows $[3,27,30]$ as well as by complex interactions with surrounding tissues $[15,20,26,34]$.

\footnotetext{
* Correspondence: hainandaousyd@163.com

State Key Laboratory of Crop Biology; Key Laboratory of Biology and Genetic Improvement of Horticultural Crops (Huanghuai Region), Ministry of Agriculture; College of Horticulture Science and Engineering, Shandong Agricultural University, Tai'an 271018, Shandong, China
}

Gravity exhibits a constant force on earth, characterized by constant direction and magnitude. Terrestrial plants have evolved under gravitational forces and have the ability to use it as the most reliable signal for regulating their growth and morphogenesis [11, 36]. The orientation of plant growth relative to the gravity vector can be determined by the gravitropic set-point angle (GSA) [7]. Auxin controls the GSA in the lateral branches of higher plants; In addition, auxin regulates the GSA by adjusting the magnitude of antigravitropic offset component during the whole development process via TIR1/AFB-Aux/IAA-ARFdependent auxin signaling within the gravity-sensing cells of the roots and shoots [29].

MYB transcription factors (TFs) participate in plant metabolism, development, and response to biotic and abiotic stresses [8]. The model experimental plant Arabidopsis thaliana expresses MYB88 and FOUR LIPS (FLP), two atypical two-repeat R2R3-MYB proteins, which can bind directly to the promoters of downstream

(c) The Author(s). 2019 Open Access This article is distributed under the terms of the Creative Commons Attribution 4.0 International License (http://creativecommons.org/licenses/by/4.0/), which permits unrestricted use, distribution, and 
genes that harbor an $[\mathrm{A} / \mathrm{T} / \mathrm{G}][\mathrm{A} / \mathrm{T} / \mathrm{G}] \mathrm{C}[\mathrm{C} / \mathrm{G}][\mathrm{C} / \mathrm{G}]$ motif but are not able to bind to the canonical R2R3MYB cis-elements ATAACGG or CC[T/A]ACC [43]. FLP and MYB88 downregulate the expression of a group of core cell cycle genes, such as CYCLINA2;3, CYCLINDEPENDENT KINASE (CDK) B1;1 and CDKA;1, by directly binding cis-regulatory elements in the promoters of these genes [32, 43, 46]. On the other hand, FLP and MYB88 have been shown to regulate female reproductive development [21], the late stages of stomatal development [16], guard mother cell proliferation [16, 43], root gravitropism [38, 44] and cold hardiness [42]. The molecular and cellular control of a lobed cell morphology has been suggested to involve PIN-FORMED (PIN)-type plasma membrane efflux carriers that generate subcellular auxin gradients [2]. Following the gravitational stimulation of primary roots, the subcellular localization of the PIN proteins [28, 39], such as PIN3 and PIN7, become repolarized, leading to a redirected auxin flux to the lower side of the root, root tip bending and differential cell elongation [10, 14, 29, 33]. The PINformed family is conducive to almost every step of these regulatory auxin fluxes $[3,22,27]$, and their distribution can predict auxin transport and explain auxin distribution patterns [1]. The main mechanism for regulating PIN expression is implicated as the canonical auxin signaling pathway [27, 35], which is defined by the auxininduced proteolysis of the transcriptional repressor of the Aux/IAA family, thus derepressing auxin response transcription factors [33]. Auxin response transcription factor7 (ARF7) and ARF7-regulated FLP/MYB124 transcription factors jointly form a coherent feed-forward motif that mediates transcription of auxin-responsive PIN3 in planta to control lateral root formation in the early steps [5].

The adventitious roots of self-rooted apple stocks are weak, as they are shallow and lack solidity, resulting in poor drought and cold resistance. The reason for this weakness is that there is a substantial difference between the development of the GSA from a self-rooted stock and a seedling rootstock. Therefore, it is of great importance to study the molecular mechanism of the establishment of the GSA in adventitious roots of self-rooted apple stocks for breeding seedlings with deep roots and increased resistance to stress. In contrast to A. thaliana, little is known about the regulation of root gravitropism in apple. In this study, we used overexpression and RNAi to study the function of an R2R3-MYB protein, MdFLP, in the formation of the GSAs of adventitious roots of self-rooted apple stocks. We show that the apple R2R3-MYB transcription factor MdFLP directly regulates transcription levels of the MdPIN3 and MdPIN10 genes, which in turn mediates auxin transport and the gravitropic response of adventitious roots of self-rooted apple stocks. Our findings provide new insights into the transcriptional regulation of $M d F L P$ by the auxin response factor MdARF19 in the regulation of the GSA of adventitious apple roots in response to gravity signals.

\section{Results}

The content of the endogenous hormone IAA and the expression pattern of MdPINs in shallow- and deeprooted adventitious roots of self-rooted apple stocks

In this study, two affinis and self-rooted apple stocks with shallow and deep roots and different adventitious root branch angles were selected and compared. These selfrooted stocks provided suitable materials for the study of the GSA of adventitious roots. We screened superior shallow-rooted and self-rooted apple stocks (13-13 and 13-10) and superior deep-rooted and self-rooted apple stocks (13-22 and 12-2). As shown in Fig. 1a-d, we investigated the content of endogenous hormones in the adventitious roots of the self-rooted apple stock and found that the IAA content in 13-22 was slightly increased compared to that in 13-13 on shoot cutting at $30 \mathrm{~d}$ and $42 \mathrm{~d}$ (Fig. 1e). The PIN-formed family contributes to nearly every step of these regulatory auxin fluxes $[3,22,27]$, and their distribution can predict auxin transport and explain auxin distribution patterns [1]. In this study, we investigated whether the expression of MdPIN3 and MdPIN10 was higher in 13-22 than in other self-rooted stocks (Fig. 1f). The results suggested that the endogenous hormone auxin and MdPINs may play an important role in the GSA of adventitious roots of self-rooted apple stocks.

\section{Expression pattern of MdFLP in the adventitious roots of self-rooted apple stocks}

In Arabidopsis, we found that MYB88 and FLP regulate PIN3 and PIN7 transcripts [38]. To further characterize the function of MdPINs, we used MdPIN3 and MdPIN10 promoter sequences as baits to perform yeastone-hybrid $(\mathrm{Y} 1 \mathrm{H})$ screening with a self-rooted apple stock cDNA library. As a result, one positive colony containing a cDNA fragment of MdFLP was obtained. The sequences of the full-length MdFLP protein, FLP and other R2R3-MYB proteins are highly conserved (Additional file 1: Figure S1a). To understand the evolutionary relationship between MdFLP and other related R2R3-MYB proteins in plant species, we also constructed a phylogenetic tree using the neighbor-joining (NJ) method [31]. MdFLP and AtFLP were found to be clustered within the same clade (Additional file 1: Figure S1b). MdFLP is closely related to AtFLP, which regulates root gravitropism, suggesting that MdFLP may be involved in the gravitropism of adventitious roots from self-rooted apple stocks.

To determine the subcellular localization of MdFLP, a translational fusion sequence of the full-length MdFLP 

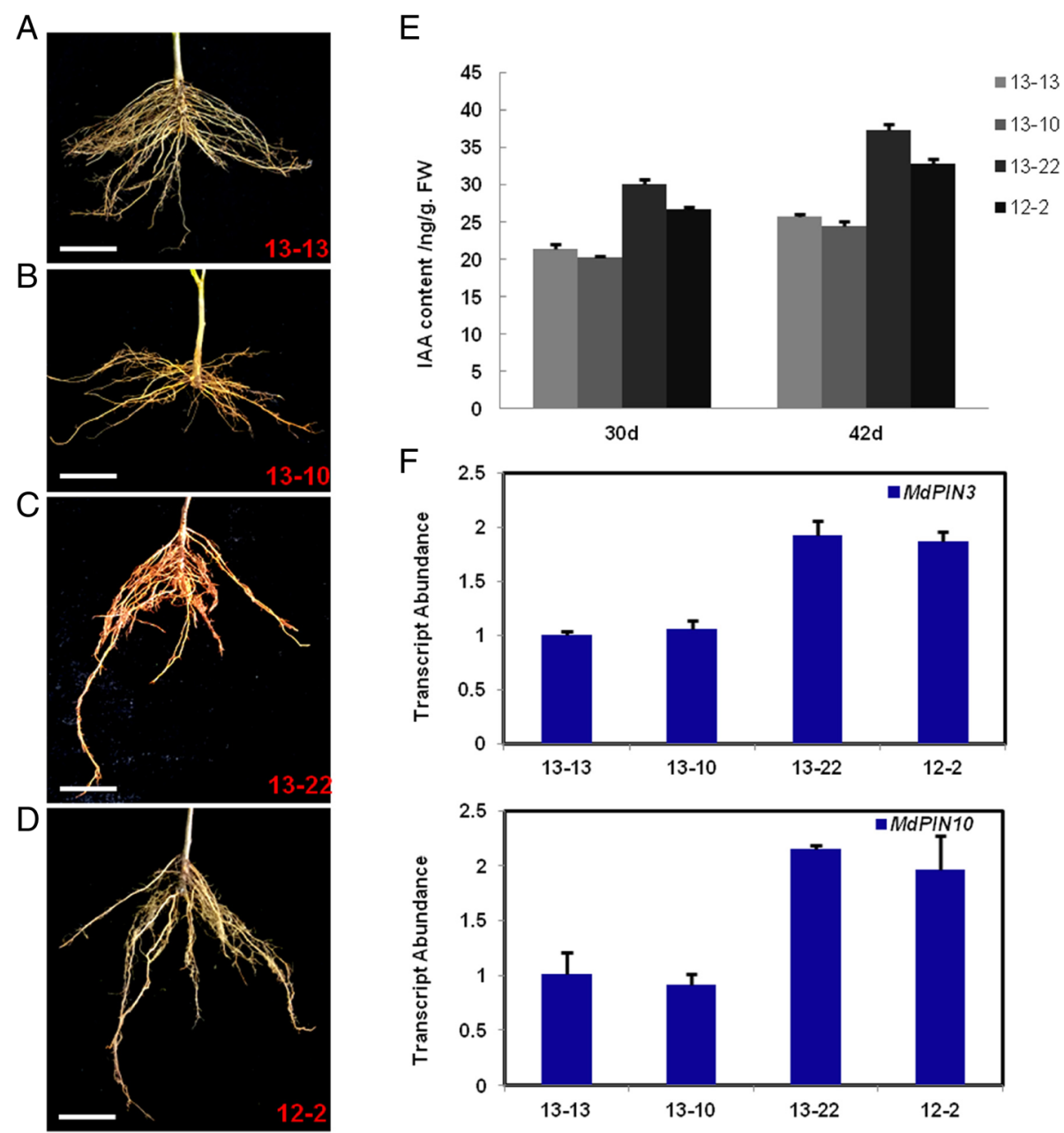

Fig. 1 The content of IAA and expression pattern of MdPINs in the adventitious roots of apple self-rooted stocks. (a) The superior shallow-rooted and self-rooted apple stock: 13-13. (b) The superior shallow-rooted and self-rooted apple stock: 13-10. (c) The superior deep-rooted and selfrooted apple stock: 13-22. (d) The superior deep-rooted and self-rooted apple stock: 12-2. (e) The content of the endogenous hormone IAA in the adventitious roots of self-rooted apple stocks. (f) Transcript levels of MdPIN3 and MdPIN10 in the adventitious roots of self-rooted apple stocks. Scale bars $=2 \mathrm{~cm}$. Error bars indicate $\pm \mathrm{SD}(n=3$, from three technical replicates). Values in Fig. 1 were derived from experiments that were performed at least three times with similar results, and representative data from one replicate are shown

coding region and the coding sequence of the green fluorescent protein (GFP) reporter under the control of the $35 \mathrm{~S}$ promoter (35S::MdFLP-GFP) was constructed. Tobacco leaf epidermal cells expressing this fusion protein showed a fluorescent signal in the nucleus, while those cells expressing the $35 \mathrm{~S}:$ :GFP control had a fluorescent signal throughout the whole cell (Fig. 2a). Given the importance of auxin in triggering lateral root development [5], we probed whether MdFLP expression was induced by auxin in the adventitious roots of self-rooted apple stocks. In auxin-treated roots, the $M d F L P$ transcripts were rapidly upregulated (Fig. 2b). To better understand the function of MdFLP, we investigated its gene expression in the adventitious roots of self-rooted apple stocks and found that the expression of MdFLP in superior deeprooted and self-rooted apple stocks increased $(\sim 2$-fold $)$ compared to that in the superior shallow-rooted and selfrooted apple stock (Fig. 2c). These results suggest that $M d F L P$ plays an important role in the gravitropism of adventitious roots of self-rooted apple stocks.

\section{MdFLP directly regulates MdPIN3 and MdPIN10 transcription}

To determine the mechanism by which MdFLP regulates the GSA of adventitious roots of self-rooted apple stocks, $\mathrm{Y} 1 \mathrm{H}$ assays were performed. Two-kb fragments upstream of the ATG start codon of MdPIN3 and MdPIN10 were cloned into the pHIS2 vector. MdFLP was inserted into the pGADT7 vector, and the pHIS2 vector was used as a control. First, we added different concentrations of 3amino-1,2,4-triazole (3-AT) to medium lacking His and Trp to determine the optimal concentrations of 3-AT that 

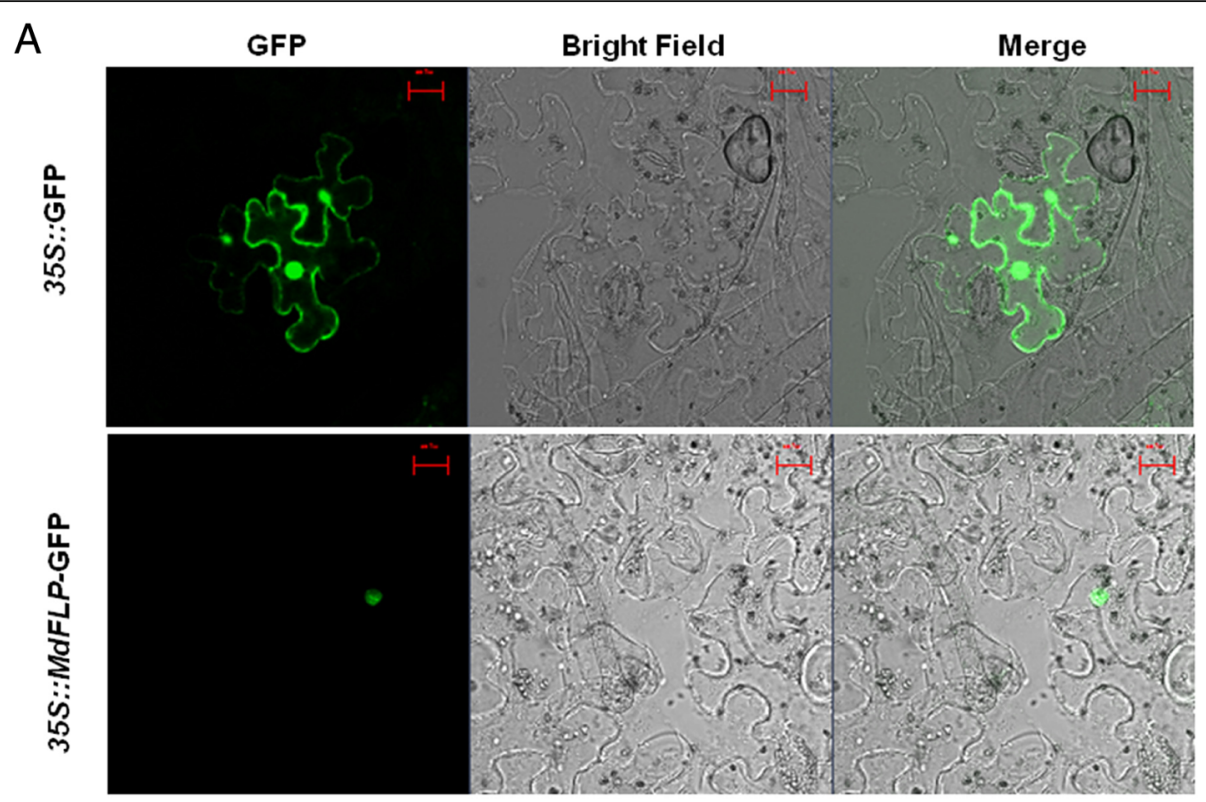

B

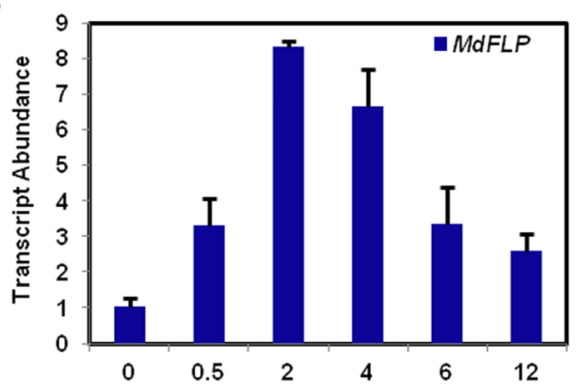

C

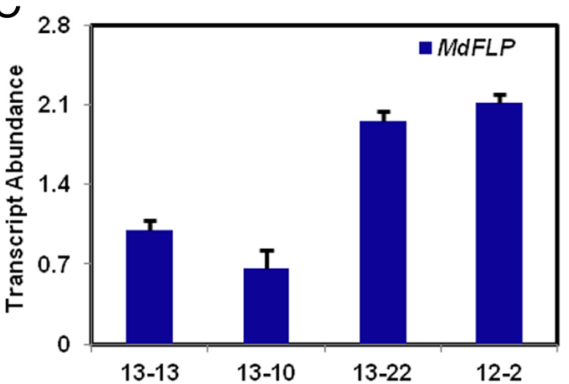

Fig. 2 MdFLP expression in response to auxin in self-rooted apple stocks and MdFLP protein localization. (a) MdFLP is localized in the nucleus of the tobacco leaf epidermal cells. GFP, green fluorescent protein. Bars, $50 \mu \mathrm{m}$. (b) qRT-PCR analysis of MdFLP expression at $0 \mathrm{~h}, 0.5 \mathrm{~h}, 2 \mathrm{~h}, 4 \mathrm{~h}, 6 \mathrm{~h}$, $12 \mathrm{~h}$ of the auxin (10 mM NAA) time course from 13 to 22 roots. (c) Transcript level of MdFLP in the adventitious roots of self-rooted apple stocks. Error bars indicate $\pm \mathrm{SD}(n=3$, from three technical replicates). Values in Fig. 2 were derived from experiments that were performed at least three times with similar results, and representative data from one replicate are shown

could suppress the background histidine leakiness of the pHIS2 vectors. This result showed that $80 \mathrm{mM}$ 3-AT was sufficient to suppress the histidine leakiness of MdPIN3 and MdPIN10. As a result, MdFLP could activate the expression of MdPIN3 and MdPIN10 (Fig. 3a). To further examine whether MdFLP triggered the transcriptional activity of the promoters of MdPIN3 and MdPIN10 in planta, a transient expression assay was performed by hypodermically injecting tobacco leaves with the MdPIN3 and MdPIN10 promoters to drive the expression of the reporter $\beta$-glucuronidase (GUS) gene. MdFLP was inserted into the pCAMBIA1300 vector. The constructs were transiently transformed into tobacco leaves, and 35S::GUS was used as a control. The results indicated that the tobacco leaves expressing proMdPIN3::GUS plus MdFLP showed much higher GUS activity than the tobacco leaves expressing the control (proMdPIN3::GUS). Additionally, the tobacco leaves expressing proMdPIN10::GUS plus MdFLP exhibited higher GUS activity than the tobacco leaves expressing proMdPIN10::GUS (Fig. 3b). Together with the results of the $\mathrm{Y} 1 \mathrm{H}$ and GUS transient expression assays, it was determined the MdFLP activated the expression of MdPIN3 and MdPIN10 in a self-rooted apple stock.

MYB transcription factors are known to regulate their downstream target genes by binding to MYB cis-elements in the promoter region and subsequently activating their expression. MdFLP acts as an MYB transcription factor that can bind directly to the promoters of downstream genes that harbor an $[\mathrm{A} / \mathrm{T} / \mathrm{G}][\mathrm{A} / \mathrm{T} / \mathrm{G}] \mathrm{C}[\mathrm{C} / \mathrm{G}][\mathrm{C} / \mathrm{G}]$ motif [43]. We analyzed the promoter sequences of MdPIN3 and MdPIN10 and found that they contain a total of five potential MdFLP binding sites, namely, AACGG, AGCC G, CGCGG, AGCCG and TACCC (Fig. 3c and Additional file 1: Figure S4). To examine whether MdFLP directly bound to these motifs in plants, we carried out a chromatin immunoprecipitation quantitative real-time PCR (ChIP-qPCR) assay. Our results showed that MdFLP bound to fragment b, e, which contains an AGCCG cis- 


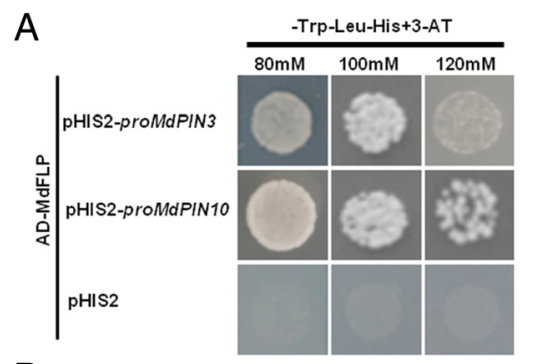

B
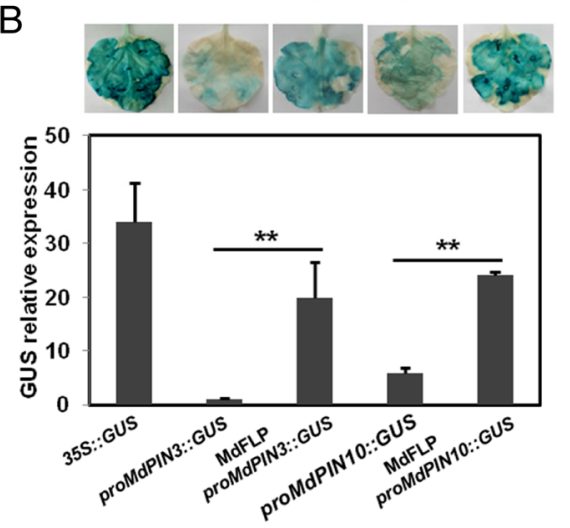

E

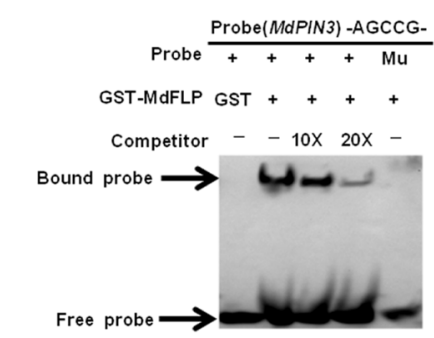

C
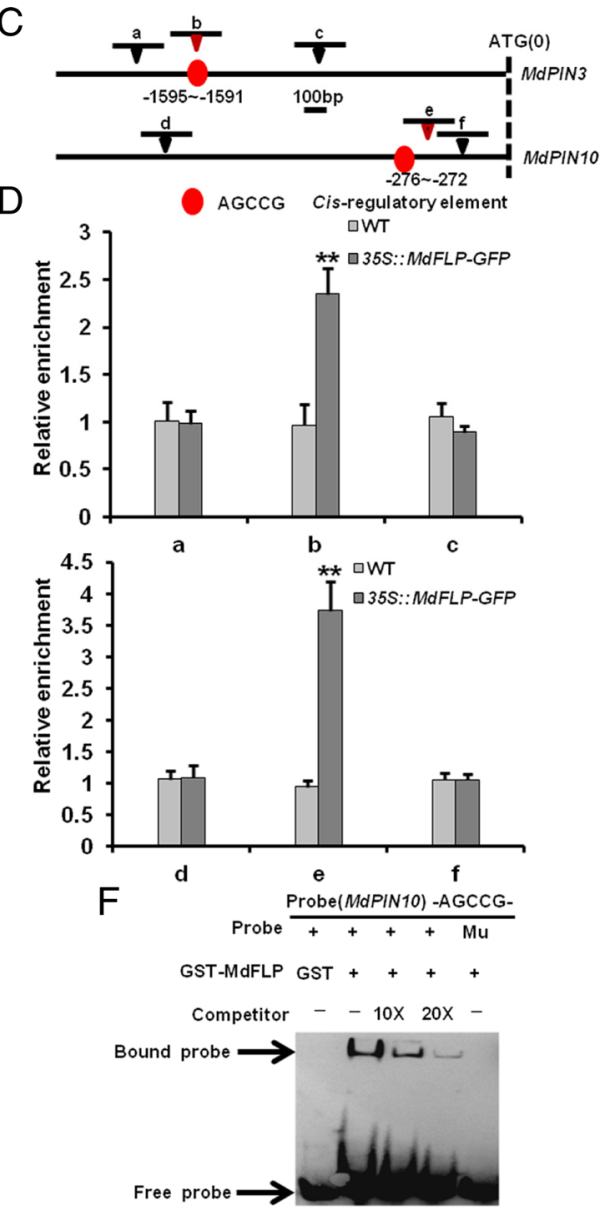

Fig. 3 MdFLP directly binds to the promoters of MdPIN3 and MdPIN10. (a) Y1H assays. MdFLP can bind to the promoter fragments of MdPIN3 and MdPIN10 in a Y1H assay. (b) GUS transient expression assays. Activation assay of MdPIN3 and MdPIN10 promoter activity in tobacco leaves by transient expression of MdFLP together with other effector genes of different combinations, as determined through a GUS activity assay. GUS relative expression level in transgenic leaves by qRT-PCR analysis is shown. At least three independent samples were used for gene expression analyses. Error bars represent \pm SE. Significant differences were determined by Student's $t$-test $\left.{ }^{* *} P<0.01\right)$. (c) Schematic diagram of the promoters in the MdPIN3 and MdPIN10 genes. Red circles indicate AGCCG. The translational start site (ATG) is shown at position 0. Fragment b, e contains the cis-element AGCCG, fragment a contains the cis-element AACGG, fragment c contains the cis-element CGCGG, fragment $\mathrm{f}$ contains the cis-element TACCC, and fragment $d$ serves as a negative control. (d) ChIP-qPCR assays were performed using 'Orin' apple calli harboring 35S::MdFLP-GFP and probed using anti-GFP antibodies. PCR products that were generated by primer pairs at position ' $b$ ', ' $e$ ' shown in $c$, which include the element AGCCG, are enriched in 35S::MdFLP-GFP transgenic calli. Error bars represent \pm SE. Significant differences were determined by Student's $t$-test (**P $<0.01)$. (e-f) EMSA showing that GST-MdFLP directly binds to the promoters of MdPIN3 and MdPIN10. The GST protein was incubated with the labeled probe in the first lane to serve as a negative control. Ten- and 20-fold excess unlabeled probes were used for competition

element in the promoters of MdPIN3 and MdPIN10 (Fig. 3d and Additional file 1: Figure S5). To further confirm the specific binding of MdFLP to the promoters MdPIN3 and MdPIN10, we performed an electrophoretic mobility shift assay (EMSA) to investigate whether the fusion protein GST-MdFLP could directly bind to a 40-bp DNA probe containing the (-1595 - 1591) AGCCG motif of the MdPIN3 promoter (Fig. 3c). GST-MdFLP bound to the AGCCG-containing DNA probe, which was outcompeted by the addition of unlabeled DNA probe. By contrast, GST-MdFLP failed to bind to a mutant probe lacking the AGCCG motif (Fig. 3e), indicating that the binding of MdFLP to the MdPIN3 promoter is dependent on the presence of the AGCCG motif. A similar assay was performed to test whether MdFLP bound the MdPIN10 promoter. The fusion protein GST-MdFLP directly bound to a 40-bp DNA probe containing the $(-276 \sim-272)$ AGCCG motif from the MdPIN10 promoter, which was outcompeted by the addition of unlabeled DNA probe (Fig. 3f). Together, these data demonstrate that MdFLP can bind directly to the MdPIN3 and MdPIN10 promoters via the AGCCG element. 


\section{MdFLP is a direct target gene of MdARF19}

The auxin signaling pathway that is central to lateral root induction depends largely upon the presence of the auxin response transcription factor7 (ARF7) in Arabidopsis [17]. We found that the expression of MdFLP and MdARF19 was induced by auxin in the adventitious roots of selfrooted apple stocks (Fig. 2b and Additional file 1: Figure S6). Then, we tested whether the auxin-inducible expression of MdFLP also depended on MdARF19, and Y1H assays were performed. The results indicated that MdARF19 activated the expression of MdFLP (Fig. 4a). Additionally, a transient expression assay was conducted. The results indicated that the tobacco leaves expressing proMdFLP:: GUS plus MdARF19 showed much higher GUS activity than the leaves expressing the proMdFLP::GUS control (Fig. 4b-d). MdFLP contains two canonical AuxREs (GAGACA) upstream of its start codon, which could be the binding sites of MdARF19 (Fig. 4e). Using Y1H assays, we found that MdARF19 interacted with AuxRE1 and AuxRE2, and these interactions were lost when the AuxRE motifs were mutated (mAuxRE) (Fig. 4f). Thus, our data demonstrate that $M d F L P$ is a direct target gene of MdARF19.

\section{MdFLP, MdARF19 and MdPIN transcripts are induced by microgravity}

To maintain normal root gravitropism, plants must receive and respond to the gravity signal appropriately [37]. We investigated the effects of a microgravity environment on the root growth and endogenous hormone IAA in 12-2. The roots that had been cultivated under artificial microgravity conditions in the orbit failed to grow toward gravity (Fig. 5a). The length of the 12-2 roots increased during the cultivation period under microgravity conditions (Additional file 1: Figure S7a-e). When we investigated the content of endogenous hormones under microgravity conditions, we found that the IAA content in WY-1 was higher than that in CKY-1 (Fig. $5 \mathrm{~b})$. This result suggested that the metabolism of IAA was perturbed by the lack of gravitational force in the adventitious roots of self-rooted apple stocks.

To investigate whether the expression of MdFLP, MdARF19 and MdPINs is induced by microgravity, we

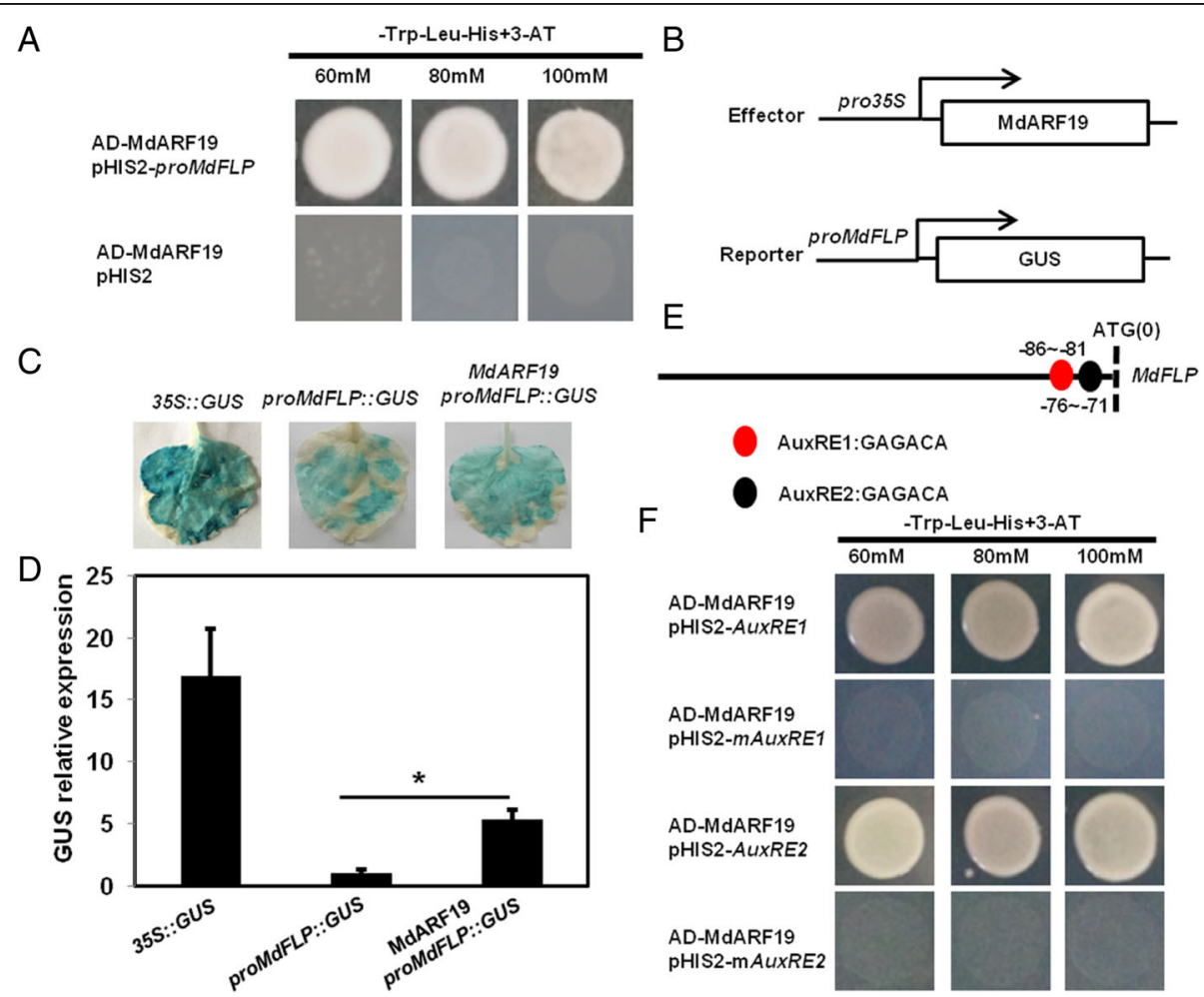

Fig. 4 MdARF19 directly binds to the promoter of MdFLP. (a) Y1H assays showed that MdARF19 can bind to the promoter fragments of MdFLP. (b) Effector constructs and the MdFLP promoter-driven reporter gene construct for transient expression assays. (c) GUS transient expression assays. Activation assay of MdFLP promoter activity in tobacco leaves by transient coexpression of MdARF19, as determined through a GUS activity assay. (d) GUS relative expression level in transgenic leaves by qRT-PCR analysis is shown. The endogenous gene RNR-2 of tobacco was used as an internal control for normalization. At least three independent samples were used for gene expression analyses. Error bars represent \pm SE. Significant differences were determined by Student's $t$-test $\left({ }^{*} P<0.05\right)$. (e) Schematic diagram of the promoter in the MdFLP gene. The red circles indicate the AuxRE1 GAGACA motif. The black circle indicates the AuxRE2 GAGACA motif. The translational start site (ATG) is shown at position 0. (f) $\mathrm{Y} 1 \mathrm{H}$ assays show that MdARF19 binds to AuxRE1 and AuxRE2 of structural MdFLP 
A
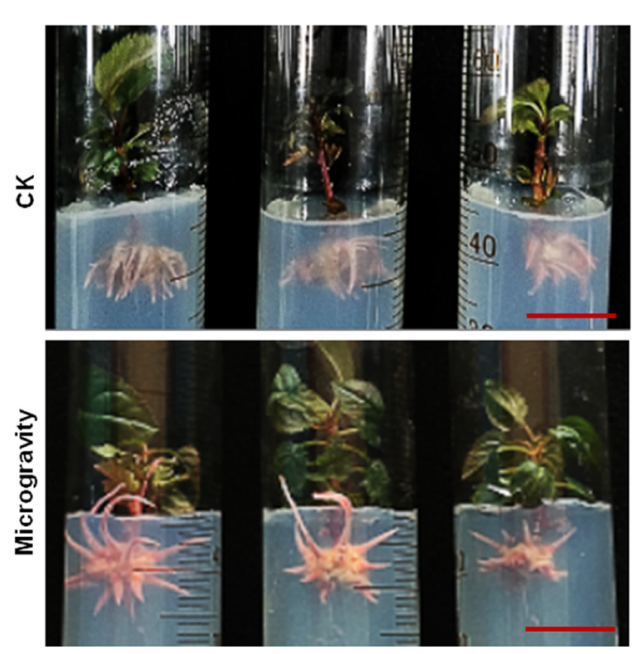

B

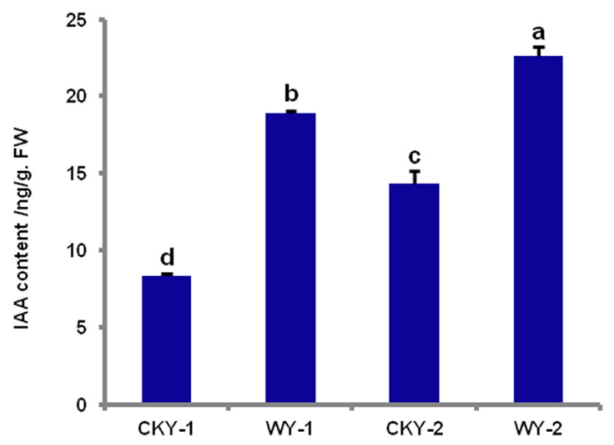

C

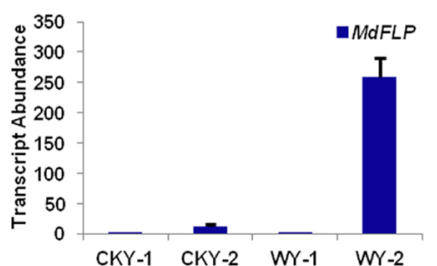

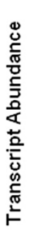

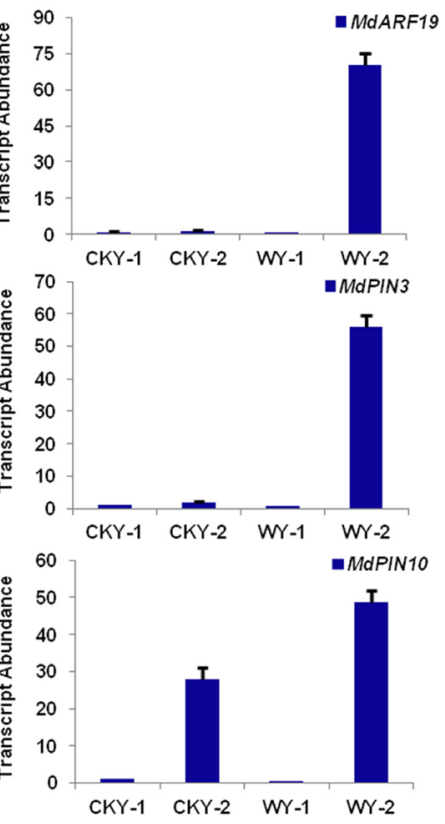

Fig. 5 Transcript levels of the gravity-responsive genes and the content of IAA in 12-2 under microgravity. (a) The adventitious roots of apple self-rooted stocks failed to grow toward gravity under microgravity. Scale bars $=1 \mathrm{~cm}$. (b) The content of the endogenous hormone IAA in the adventitious roots of apple self-rooted stocks under microgravity. Samples with different letters are significantly different: $P<0.05$ (Fisher's LSD mean separation test). (c) Transcript levels of endogenous gravity-responsive genes of MdFLP, MdARF19, MdPIN3 and MdPIN10 in apple stock in vitro (12-2). CKY-1 indicates primary adventitious roots (7 d), CKY-2 indicates primary adventitious roots (14 d), WY-1 indicates primary adventitious roots under microgravity (7 d), and WY-2 indicates primary adventitious roots under microgravity (14 d). Error bars indicate \pm SD ( $n=3$, from three technical replicates). Values in (c) were derived from experiments that were performed at least three times with similar results, and representative data from one repetition are shown

used quantitative real-time RT-PCR to evaluate their expression under microgravity. As shown in Fig. 5c, MdFLP expression was detected in all examined stages, with the highest levels in WY-2. The transcript levels of MdARF19, MdPIN3 and MdPIN10 were also analyzed in different stages of the adventitious roots of self-rooted apple stocks under microgravity and were expressed at higher levels in WY-2 than in CKY-1 or CKY-2. In addition, we detected two homologs of MdoMYB148/MdFLP, MdARF7/ $M d A R F 19$, that showed similar expression patterns (Additional file 1: Figure S7f). These results suggested that as a key factor, $M d F L P$ responds to gravity during the gravitropism of adventitious roots of self-rooted apple stocks.

\section{MdFLP determines the GSA of the adventitious roots of self-rooted apple stocks via MdPIN3 and MdPIN10}

To assess the roles of MdFLP in regulating the expression of MdPIN3 and MdPIN10 in adventitious roots, we compared the GSAs of adventitious roots in several transgenic lines. We constructed the 35S::MdFLP, 35S::MdPIN3, 35S::MdPIN10, and MdFLP-RNAi vectors, which were transiently transformed into the root cells of 'Gala' via Agrobacterium rhizogenes-mediated genetic transformation, meanwhile an empty vector was used as a control. The 35S::MdFLP (OX-1, OX-2 and OX-3), 35S::MdPIN3 (OX-2, OX-4 and OX-7) and 35S::MdPIN10 (OX-1, OX-5 and OX-8) transcript levels were markedly higher than the empty vector control transcript levels. In parallel, the MdFLP-RNAi (\#1, \#3 and \#7) transcript levels were markedly lower than the empty vector control transcript levels, indicating that chimeric apples composed of wild-type (WT) shoots and transgenic roots were successfully obtained (Fig. 6f). Three batches of 35S::MdFLP chimeric transgenic apples denoted as OX-1, OX-2 and OX-3, similar to 35S::MdPIN3, 35S::MdPIN10, and MdFLP-RNAi, were evaluated. During the emergence of adventitious roots from the self-rooted apple stocks, MdFLP (OX-1, OX-2 and OX-3) (Fig. 6b), similar to MdPIN3 (OX-2, OX- 

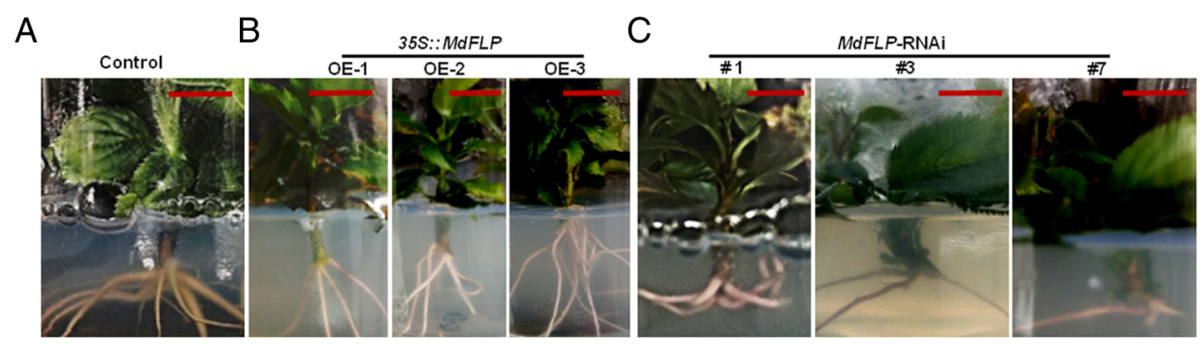

D

35S::MdPIN3

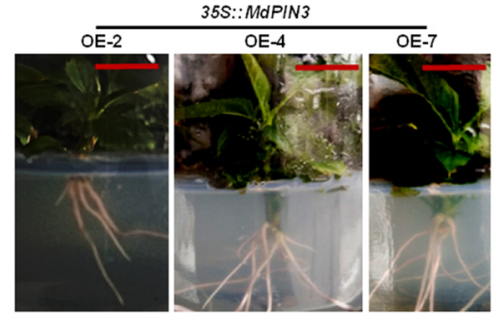

E

E

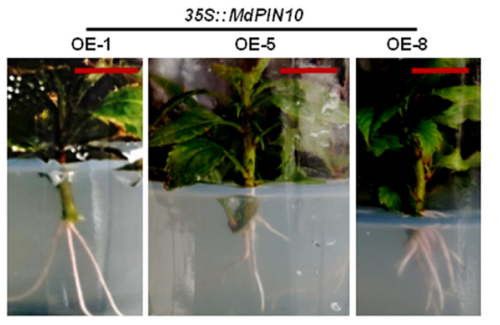

$\mathrm{F}$
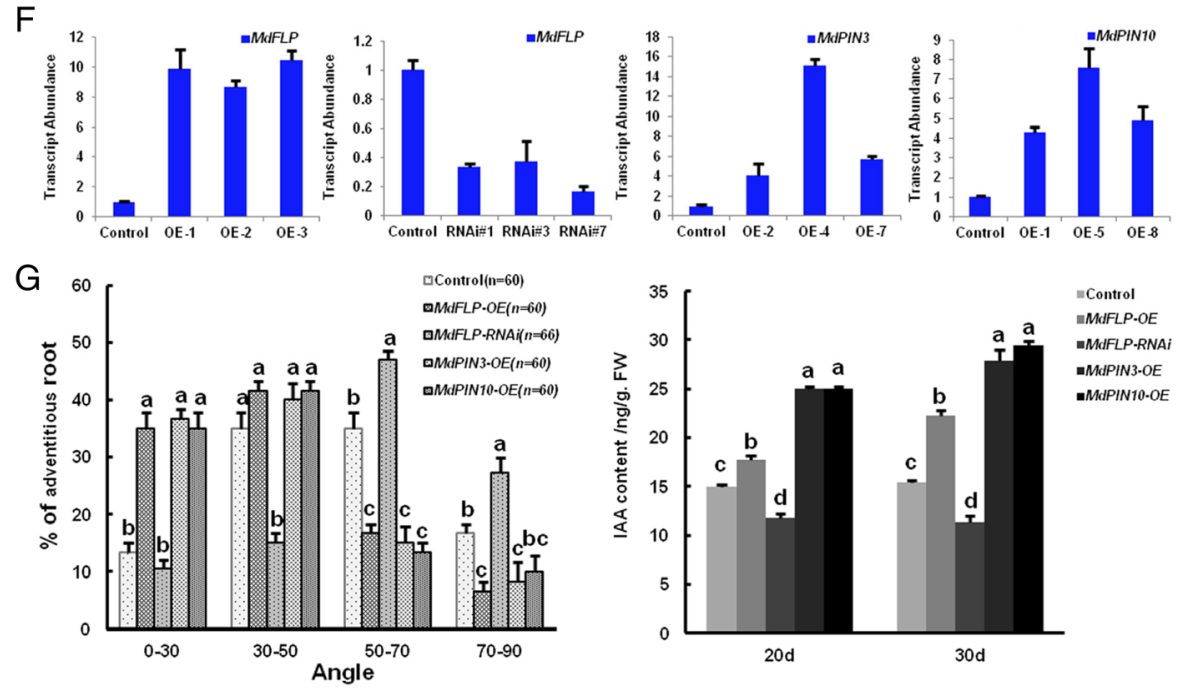

Fig. 6 Function of MdFLP in setting the GSA of adventitious roots. (a-e) The gravitropic response of adventitious roots was measured by the angles between empty vector controls and transgenic lines. Scale bars $=1 \mathrm{~cm}$. 35S::MdFLP adventitious roots (b), similar to 355::MdPIN3 (d) and 35S::MdPIN10 (e), display a smaller GSA than the empty vector controls (a). MdFLP-RNAi adventitious roots (c) have a larger GSA than empty vector controls. (f) Transcript levels of endogenous gravity-responsive genes in transgenic plant roots. Error bars indicate $\pm S D(n=3$, from three technical replicates). Values in (f) were derived from experiments that were performed at least three times with similar results, and representative data from one replicate are shown. (g) Distributions of adventitious root GSAs. Similar to 35S::MdFLP, 35S:::MdPIN3 and 35S::MdPIN10 show stronger gravitropic responses than the empty vector controls, resulting in a smaller GSA. However, MdFLP-RNAi displays a larger GSA than that of the empty vector controls. Samples with different letters are significantly different: $P<0.05$ (Fisher's LSD mean separation test). (h) The content of IAA in the roots of transgenic lines after $20 \mathrm{~d}$ and $30 \mathrm{~d}$. Similar to 35S::MdFLP, 355::-MdPIN3 and 35S::MdPIN10 show higher IAA content than the control. However, MdFLP-RNAi displays a lower IAA content than the control. Samples with different letters are significantly different: $P<0.05$ (Fisher's LSD mean separation test)

4 and OX-7) and MdPIN10 (OX-1, OX-5 and OX-8) (Fig. 6d-e), grew downwards faster and exhibited a smaller GSA than the empty vector controls. For example, over $20 \%$ of the MdFLP-OX adventitious roots increased within a $0-30^{\circ}$ range, while over $19 \%$ of the adventitious roots fell within a $50-70^{\circ}$ range. Notably, similar to MdFLP-OX, MdPIN3-OX and MdPIN10-OX adventitious roots exhibited a reduced GSA, with more roots displaying GSAs within $0-30^{\circ}$ (Fig. 6g). In contrast, MdFLPRNAi displayed a larger GSA than that of the empty vector controls (Fig. 6c), with more roots displaying GSAs within $50-70^{\circ}$ (Fig. 6g).

In addition, MdPIN3 and MdPIN10 expression was detected in $M d F L P$ transgenic roots (Additional file 1: Figure S8). The transcripts were remarkably upregulated in 35S:: MdFLP and remarkably downregulated in MdFLP-RNAi compared with those in the empty vector controls, indicating that $M d F L P$ functions in controlling adventitious root bending via MdPIN3 and MdPIN10. Then, we investigated the content of IAA in transgenic line roots and 
found that the content of the endogenous hormone IAA in $355:: M d F L P$ roots under $20 \mathrm{~d}$ and $30 \mathrm{~d}$ was higher than that in the adventitious roots of the control, similar to 35S::MdPIN3 and 35S::MdPIN10. Therefore, the increased level of IAA in these transgenic roots likely contributes to sustaining the rapid elongation of apple roots. In contrast, $M d F L P$-RNAi displayed a lower IAA content than the adventitious roots of the control (Fig. 6h). Together, these results demonstrate that MdFLP functions in setting the GSA of adventitious roots of self-rooted apple stocks via MdPIN3 and MdPIN10.

\section{Gravity is essential for MdFLP to determine the GSA of the adventitious roots of self-rooted apple stocks}

Since gravity profoundly influences root growth and development, plants respond to changes in orientation by using gravitropic responses to modify their growth. We found that the expression of MdFLP was induced by microgravity. To investigate whether gravity is essential for MdFLP to determine the GSA of adventitious roots of self-rooted apple stocks, we observed the phenotype of the 35S::MdFLP roots under microgravity. The MdFLP (WOX-1, WOX-2) and MdFLP (OX-7, OX-9) transcripts were remarkably upregulated compared with the empty vector control transcripts in the roots of these apples, indicating that chimeric apples composed of WT shoots and transgenic roots were successfully obtained (Fig. 7b). During the growth and development of transgenic roots under microgravity, MdFLP (WOX-1, WOX-2) adventitious roots grew faster and displayed a larger GSA than the adventitious roots of the empty vector controls and MdFLP (OX-7, OX-9) under normal conditions (Fig. 7a). For example, over $20 \%$ of MdFLP-OX-W adventitious roots increased within a $70-90^{\circ}$ range, while over $30 \%$ of adventitious roots fell within a $30-50^{\circ}$ range for MdFLPOX (Fig. 7c). In contrast, 35S::MdFLP had a smaller GSA than the empty vector controls under normal conditions, indicating that MdFLP functions in controlling adventitious root bending via gravity. We then investigated the content of IAA in transgenic line roots and found that the content of the endogenous hormone IAA in MdFLP-OXW roots after $20 \mathrm{~d}$ and $30 \mathrm{~d}$ was higher than that in the control and MdFLP-OX roots (Fig. 7d). Therefore, the higher level IAA in of the MdFLP-OX-W roots likely contributes to the rapid elongation of apple roots. In conclusion, these results demonstrate that MdFLP requires gravity to set the GSA of adventitious roots of self-rooted apple stocks.

\section{Discussion}

MdFLP is involved in the gravitropism of adventitious roots from self-rooted apple stocks

We have shown that FLP is an angle-inducible gene required for gravity and angle-responsive gene expression in apples. We cloned MdFLP from Malus $\times$ domestica. An analysis of the MYB proteins from Malus $\times$ domestica cataloged by the Genome Database for Rosaceae (GDR) was performed, and MdoMYB148 was identified [4, 9, 13]. The MdFLP transcript characterized in this study was slightly shorter than that of MdoMYB148, resulting from the inclusion of an alternative exon in the latter sequence (Additional file 1: Figure S2). Comparison of the nucleotide sequence of $M d F L P$ with those of MdMYB88 and MdMYB124 revealed that the sequences were highly similar (Additional file 1: Figure S3). We designated our transcript as MdFLP to distinguish it from previously identified sequences.

The disruption of auxin transport such as in pin2,3,4,7 multiple mutants leads to abnormal patterns and excess stomatal production. The preferential expression of PIN3 in stomatal precursors is closely related to dynamic cellular auxin activities, as well as differentiation processes and cell fate [18]. It has previously been shown that FLP and MYB88 play a redundant role during the last division in the stomatal pathway by regulating the transcription of core cell cycle genes [32, 43, 46]. As pleiotropic transcription factors, FLP and MYB88 regulate various plant development processes and response to environmental changes $[21,44]$. Apple MdFLP is functionally conserved with Arabidopsis FLP, at least for root gravitropism, because expression of MdFLP in transgenic plants resulted in a smaller or larger GSA than the expression of the empty vector controls (Fig. 6a-c). Two lines of evidence support the roles of MdFLP in the gravitropism of adventitious roots of apple. First, transgenic apple plants expressing 35S:MdFLP displayed a smaller GSA than apple plants expressing the empty vector controls (Fig. 6a-b). Second, $M d F L P$-RNAi plants displayed a larger GSA than plants harboring empty vector controls (Fig. 6c). In addition to their roles in the gravitropism of adventitious roots of apple, we also observed that 35S::MdPIN3 and 35S:: MdPIN10 (Fig. 6d-e) grew downwards faster and exhibited a smaller GSA than the empty vector controls. MdFLP influenced the GSA during the process of root gravitropism. This process may be due to the basal expression of angleresponsive genes in transgenic plants, including MdPIN3 and MdPIN10 (Fig. 6).

\section{Gravity is essential during the formation of the GSA of adventitious roots of self-rooted apple stocks}

Plant gravitropic responses consists of three phases: sensing, signal transduction and asymmetric organ growth. DR5 signals and quantitative analysis of differential DIIVENUS revealed that the delayed gravitropic response of $f l p$ was related to reduced auxin asymmetry across roots [38]. Since gravity profoundly influences root growth and development, plants respond to changes in orientation by using gravitropic responses to modify their growth. The 

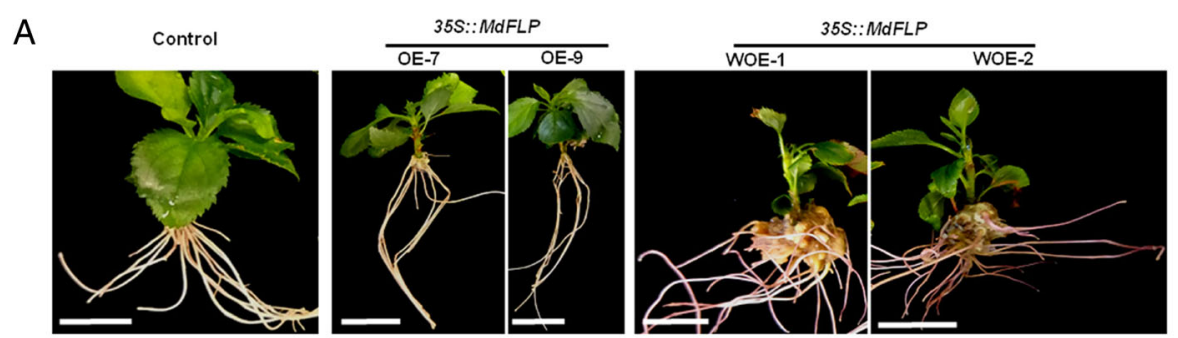

B
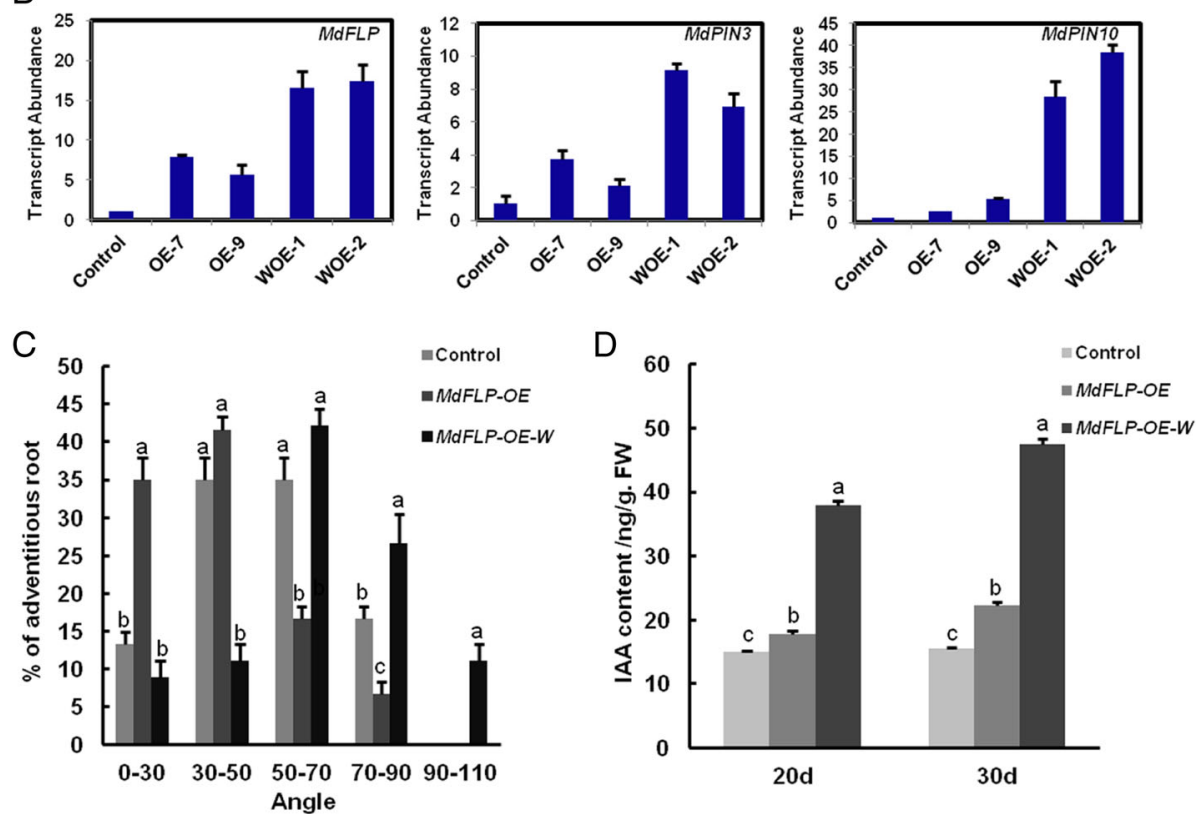

Fig. 7 The gravity signal plays a key role in setting the adventitious root GSA. (a) The gravitropic response of adventitious roots was measured by the angles between empty vector controls. Scale bars $=1 \mathrm{~cm}$. 355::MdFLP and 35S::MdFLP under microgravity. 355::MdFLP adventitious roots display a smaller GSA than the adventitious roots of the empty vector control. However, 35S::MdFLP adventitious roots under microgravity have a larger GSA than the adventitious roots of the empty vector control and 35S::MdFLP. (b) Transcript levels of endogenous gravity-responsive genes in empty vector controls, 35S::MdFLP and 355::MdFLP under microgravity. Error bars indicate \pm SD ( $n=3$, from three technical replicates). Values in (b) were derived from experiments that were performed at least three times with similar results, and representative data from one replicate are shown. (c) Distributions of adventitious root GSAs. 35S::MdFLP show stronger gravitropic responses than the empty vector control, resulting in a smaller GSA. However, the 355::MdFLP adventitious roots under microgravity have a larger GSA than the empty vector control and 355::MdFLP adventitious roots. Samples with different letters are significantly different: $P<0.05$ (Fisher's LSD mean separation test). (d) The content of IAA in empty vector controls, 355::MdFLP and 35S::MdFLP under microgravity. The 355::MdFLP adventitious roots under microgravity showed higher IAA content than the adventitious roots of the empty vector control and 355::MdFLP. Samples with different letters are significantly different: $P<0.05$ (Fisher's LSD mean separation test)

gravitational signal greatly modifies the expression of a wide range of genes [23, 25]. Genes related to the organization and functions of microtubules are included among those whose expression is altered under different gravity conditions.

In this study, we found that the expression of MdFLP was induced by microgravity. The roots that had been cultivated under artificial microgravity conditions in the orbit failed to grow toward gravity (Fig. 5a). These results suggested that as a key factor, MdFLP responds to gravity during the establishment of the GSA of adventitious roots of self-rooted apple stocks. To investigate whether gravity is essential for MdFLP to determine the
GSA of adventitious roots of self-rooted apple stocks, the 35S::MdFLP transcripts under microgravity and normal conditions were examined and were remarkably upregulated compared with those of the empty vector control transcripts (Fig. 7b). During the growth and development of transgenic roots under microgravity, 35S:: MdFLP adventitious roots grew faster and displayed a larger GSA than the empty vector control and 35S:: $M d F L P$ adventitious roots under normal gravity (Fig. 7a) - In contrast, 35S::MdFLP adventitious roots had a smaller GSA than empty vector control adventitious roots under normal gravity, indicating that MdFLP functions in controlling adventitious root bending via gravity. 
We also found that the content of the endogenous hormone IAA in $35 S:: M d F L P$ roots was higher than that in the adventitious roots of the control. In contrast, MdFLP-RNAi displayed a lower IAA content than the adventitious roots of the control (Fig. 6h). Whether MdFLP is involved in the feed-forward transcriptional regulation of auxin homeostasis or signaling in apples requires further investigation.

The MdFLP gene is involved in a regulatory network controlling the gravitropism of adventitious roots of apple via MdPIN3 and MdPIN10

The approach of systematic evolution of ligands by exponential enrichment (SELEX) was used, and $[\mathrm{A} / \mathrm{T} /$ $\mathrm{G}][\mathrm{A} / \mathrm{T} / \mathrm{G}] \mathrm{C}[\mathrm{C} / \mathrm{G}][\mathrm{C} / \mathrm{G}]$ was identified as a distinct binding motif for MYB88/FLP [43]. MdFLP shares a relatively high sequence similarity with FLP, and MdFLP functions in setting the GSA of adventitious roots of self-rooted apple stocks (Fig. 6). Our ChIP-qPCR and EMSA experiments revealed that MdFLP was able to bind to AGCCG. Moreover, ChIP-chip analysis was also carried out in Arabidopsis with anti-MYB88/FLP antibodies and identified potential targets of $c .226$, encoding proteins involved in the cell division, cell cycle, oxidative stress, DNA binding, cold stress tolerance, splicing, etc. [43]. We also identified the MdFLP gene with roles in hormone response, suggesting that the function of MdFLP might be more diverse than that of the FLP gene in Arabidopsis.

MdFLP functions in setting the apple self-rooted stock adventitious root GSA via MdPIN3 and MdPIN10 (Fig. 6 and Additional file 1: Figure S8). In Arabidopsis, we also found that MYB88 and FLP regulate PIN3 and PIN7 transcripts [38]. Moreover, Chen et al. [5] found that ARF7 also controls auxin-sensitive FLP expression, thus defining a coherent FFM for auxin-induced PIN3 expression. In our study, using $\mathrm{Y} 1 \mathrm{H}$ assays, we found that MdARF19 interacted with AuxRE1 and AuxRE2, and these interactions were lost when the AuxRE sequences were mutated (mAuxRE) (Fig. 4f). Thus, our data demonstrate that MdFLP is a direct target of MdARF19. The involvement of MdARF19 in the feed-forward transcriptional regulation of $M d F L P$ during the formation of the GSA of adventitious roots of self-rooted apple stocks needs further investigation.

Based on the data presented in this work, we propose a model for the function of MdFLP in the gravitropic response of adventitious roots of self-rooted apple stocks (Fig. 8). With the gravity signal, MdFLP acts upstream of MdPIN3, and MdPIN10 directly regulates their expression by binding to the AGCCG motif of their promoters. Additionally, the apple auxin response factor MdARF19 influences the expression of those auxin efflux carriers and the establishment of the GSA of adventitious roots

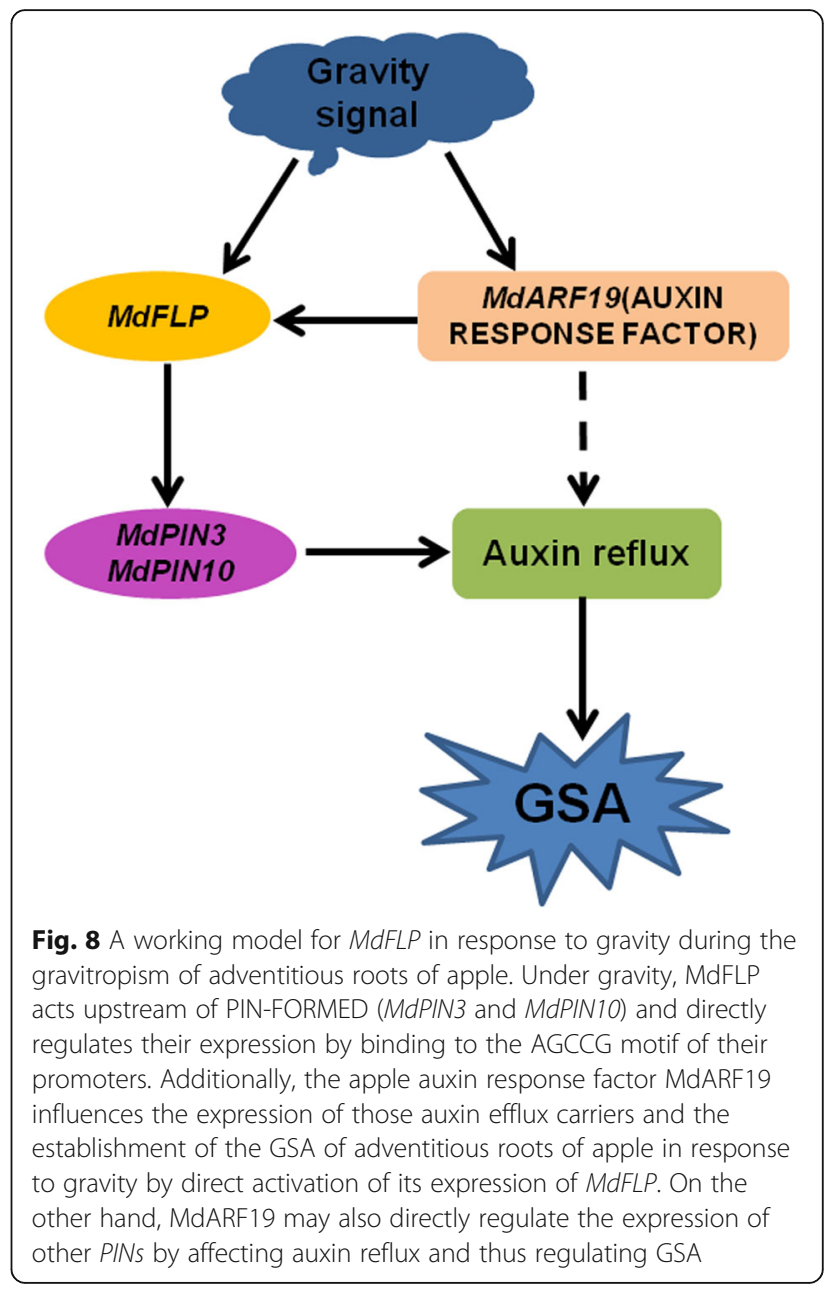

of apple in response to gravity by direct activation of its expression of MdFLP. Overall, our findings provide new insights into the transcriptional regulation of $M d F L P$ by the auxin response factor MdARF19 in the regulation of the GSA of adventitious roots of self-rooted apple stocks in response to gravity.

\section{Conclusions}

Taken together, MdFLP directly binds to the promoters of MdPIN3 and MdPIN10, activating their transcriptional expression and thereby promoting the development of adventitious roots of self-rooted apple stocks. Additionally, MdARF19 influences the expression of those auxin efflux carriers and the establishment of the GSA of adventitious roots of apple in response to gravity by direct activation of the expression of MdFLP. Overall, our findings provide new insights into the transcriptional regulation of MdFLP by MdARF19 in the regulation of the GSA of adventitious roots of self-rooted apple stocks in response to gravity. 


\section{Methods}

\section{Plant materials and growth conditions}

We screened shallow-rooted and deep-rooted superior self-rooted apple stocks: '13-13,' '13-10,' '13-22' and '122' (hybrid seedlings of Ralls and Malus spectabilis). The seedlings of Ralls were obtained from Shandong Institute of Pomology, and the seedings of Malus spectabilis were obtained from Botanical Garden (Beijing). The roots of the apple stock '13-22' were used for gene cloning. The shoot cultures of apple (Malus $\times$ domestica 'Royal Gala') were grown on MS subculture medium containing 0.5 mg/L 6-BA, $0.2 \mathrm{mg} / \mathrm{L}$ NAA (1-naphthylacetic acid), and $0.1 \mathrm{mg} / \mathrm{L}$ gibberellin (GA) for Agrobacterium rhizogenesmediated transformation and other analyses. Apple calli of the 'Orin' cultivar were subcultured on MS medium supplemented with $1.5 \mathrm{mg} / \mathrm{L} 2,4-\mathrm{D}$ and $0.4 \mathrm{mg} / \mathrm{L} 6-\mathrm{BA}$ at $25^{\circ} \mathrm{C}$ for $21 \mathrm{~d}$ in the dark.

\section{Sequence alignments and phylogenetic analysis}

A 1401-bp PCR fragment containing the complete $M d F L P$ coding sequence was amplified from the roots of '13-22' cDNA using primers MdFLP-F and MdFLP-R (Additional file 2: Table S1). Sequence alignment and phylogenetic analysis was performed based on R2R3MYB protein sequences from other species, retrieved using BLAST analysis in the NCBI nucleotide database (http://www.ncbi.nlm.nih.gov/nucleotide/), with the deduced amino acid sequence of MdFLP. The multiple sequence alignment of MdFLP and related R2R3-MYB proteins was performed as previously reported by Cao et al. [4]. The phylogenetic tree was constructed using the Neighbor-Joining (NJ) method with the Poisson model and 1000 bootstrap replicate tests, using the MEGA5 software [31].

\section{RNA extraction and qRT-PCR analysis}

Total RNA was extracted from specified tissues using Total RNA Isolation System. One $\mu \mathrm{g}$ of total RNA was used to synthesize cDNA with the PrimeScript ${ }^{\bullet} 1$ st Strand cDNA Synthesis Kit (Takara, Japan). qRT-PCR was performed using the SYBR green detection protocol (Takara) with an Applied Biosystems 7500 real-time PCR system (Applied Biosystems). Expression data were normalized to those of the $18 S$ gene. The experiments were performed using three biological samples with three technical repeats for each RNA extract. Expression data were presented as relative units after normalization to the reference gene as the internal control, per the $2^{-\triangle \Delta C T}$ method [19]. All primers used in this study are listed in Additional file 2: Table S1.

\section{Clinostat simulates microgravity conditions}

Clinostat, as a useful simulator of microgravity effects, has been used widely in plant biology research. We designed a monoaxial clinostat to simulate microgravity
[12]. The tissue culture seedlings were cultivated in the clinostat at $5 \mathrm{rpm} / \mathrm{min}$.

\section{Yeast one-hybrid assays}

$\mathrm{Y} 1 \mathrm{H}$ assays were performed using yeast strain Y187 (Clontech) according to the manufacturer's instructions. The MdFLP and MdARF19 genes were cloned into the pGADT7 vector to generate the construct AD-MdFLP, AD-MdARF19. The promoter fragments of MdFLP, MdPIN3, MdPIN10. etc., were inserted into the pHIS2 vector. Different combinations were co-transformed into yeast Y187, and the interactions were examined on medium lacking Trp, Leu and His (SD/-Trp-Leu-His) with an optimal concentration of 3-AT. Empty vector pHIS2 was used as a negative control.

\section{Transient expression assays}

Transient expression assays were conducted using tobacco leaves. The promoters of MdFLP, MdPIN3 and MdPIN10 were inserted into pCAMBIA1300-GUS plasmid. The ORFs of MdFLP and MdARF19, were inserted into pCAMBIA1300 vector, which formed 35S::MdFLP and 35S:: MdARF19 transformants. The resultant recombinant plasmids pMdFLP::GUS, pMdPIN3::GUS, and pMdPIN10::GUS were injected into tobacco leaves with different transformants via an agrobacterium-mediated method, respectively. The injected tobacco grew 2-3 days under normal conditions. The strength of the regulation were revealed by GUS staining and GUS expression quantity.

\section{Electrophoretic mobility shift assay}

EMSA was conducted according to Xie et al. [40]. MdFLP was inserted into the pGEX4T-1 plasmid. The MdFLP-GST plasmid was expressed in BL21 and purified using glutathione Sepharose beads. Three oligonucleotide probes of the MdPIN3 and MdPIN10 promoters were labeled using an EMSA probe biotin labeling kit (Beyotime). The mutant probes were labeled and contained one mutated nucleotide. The EMSAs were conducted following the manufacturer's instructions (Thermo Scientific). The binding specificity was also examined by competition with a fold excess of unlabeled oligonucleotides. The primers used for EMSA are listed in Additional file 2: Table S1.

\section{Chromatin immunoprecipitation (ChIP)-PCR analysis}

ChIP analysis was performed with the Chromatin Immunoprecipitation Assay Kit (Millipore, MA, USA) in accordance with the protocol of the manufacturer's instructions. Protein-DNA was cross-linked for $10 \mathrm{~min}$ under a vacuum in a cross-link buffer, as described by Xie et al. [41]. Cross-linked samples were incubated in $100 \mathrm{mM}$ Gly for $5 \mathrm{~min}$ under a vacuum, thoroughly washed in double-distilled water, and frozen in liquid 
nitrogen. After resuspension in lysis buffer, the purified nuclei were thenunderwent ultrasonic treatment (five times, $3 \mathrm{~s}, 40 \mathrm{~s})$ to yield chromatin fragments of 300 to 500 bps. The extract was incubated with GFP antibodies (Beyotime, China). IP protein-DNA complexes were precipitated by protein A-Sepharose beads $\left(1.5 \mathrm{~h}, 4^{\circ} \mathrm{C}\right)$.

After IP, the DNA fragments in the IP complex were released by incubating samples overnight at $65^{\circ} \mathrm{C}$ in an elution buffer (1\% SDS, $0.1 \mathrm{M} \mathrm{NaHCO} 3$, and $0.25 \mathrm{mg} /$ $\mathrm{mL}$ proteinase $\mathrm{K})$. As an input control, a portion of sonicated, cross-linked and precleared DNA was treated accordingly, except for undergoing an IP. Finally, IP DNA was quantified using quantitative real-time PCR, with three sets of primers spanning the upstream promoter, the candidate motif and the coding region. Primers are listed in Additional file 2: Table S1.

\section{Vector construction and genetic transformation}

The full-length coding region of MdFLP, MdPIN3 and MdPIN10 were amplified and inserted into the pCXSNGFP vector [6] under the control of the $35 \mathrm{~S}$ promoter. To generate the MdFLP-RNAi construct, two fragments of MdFLP were generated through PCR amplification. These two fragments were inserted in pUCCRNAi vector, then the reverse orientation fragment was inserted into the pCAMBIA1300 vector to form the final construct 35S-MdFLP-RNAi. The ORFs of MdFLP, was inserted into PCAMBIA1300 vector, which formed 35S:: MdFLP transformant. Subsequently, the MdFLP-GFP vector was genetically transformed into apple calli with the Agrobacterium tumefaciens strains LBA4404.

Agrobacterium rhizogenes-mediated transformation was performed as described by Zhou et al. [49] with minor modifications. The 35S::MdFLP, 35S-MdFLP-RNAi, 35S:: MdPIN3 and 35S::MdPIN10 vectors were introduced into A. rhizogenes MSU440 and then used for transient transformation. Subsequently, 3-week-old 'Gala' apple tissue cultures were excised from a portion of the stem and then immersed in A. rhizogenes MSU440 solution for 15-20 min. Finally, inoculated apples were transferred to $1 / 2 \mathrm{MS}$ medium containing $300 \mathrm{mg} / \mathrm{L}$ cefotaxime at $25^{\circ} \mathrm{C}$ for adventitious root induction.

\section{Quantification of plant hormones}

Adventitious roots of apple transgenic lines and selfrooted apple stocks were excised, immediately frozen in liquid nitrogen and kept at $-80^{\circ} \mathrm{C}$ until use. Subsequently, plant hormones were measured by enzymelinked immunosorbent assay (ELISA), as described by Zhang et al. [47].

\section{Gravity set-point angle measurement}

Apple transgenic roots were used for GSA measurement. Individual GSA values were sorted into the following categories: $0-30^{\circ}, 30-50^{\circ}, 50-70^{\circ}, 70-90^{\circ}$ for GSA. Experiments were repeated independently three times. Fisher's LSD mean separation test was employed for each category. The GSA measurement was using Image J software.

\section{Accession numbers}

Sequence data from this article can be found in GDR and NCBI are listed in Additional file 2: Table S2.

\section{Additional files}

Additional file 1: Supplementary Figure S1 to S8. (PDF 2512 kb)

Additional file 2: Supplementary Tables S1 to S2. (PDF 65 kb)

\section{Abbreviations}

ARF19: Auxin response factor 19; ChIP-PCR: Chromatin immunoprecipitation (ChIP)-PCR; EMSA: Electrophoretic mobility shift assay; FLP: FOUR LIPS; GFP: green fluorescent protein; GSA: gravitropic set-point angle; GST: glutathione S-transferase; GUS: $\beta$-glucuronidase; IAA: indole-3-acetic acid; LR: Lateral root; MS: Murashige and Skoog; PIN: PIN-FORMED; qRTPCR: quantitative reverse transcription-PCR; TFs: transcription factors; WT: wild-type; Y1H: yeast one-hybrid

\section{Acknowledgments}

We thank Dr. Xuesen Chen for the shoot cultures of apple (Malus $\times$ domestica 'Royal Gala') and apple calli of the 'Orin' cultivar.

\section{Authors' contributions}

ZHW and XS designed the experiments. ZHW performed most of the experiments, and ZHW wrote the paper. JLL helped with the EMSA assay; JLL, YFM, MMZ, RW, YLH and ZQM helped with the phenotypic observations and the paper revision. XS agrees to serve as the author responsible for contact and ensures communication. All authors read and approved the final manuscript.

\section{Funding}

This work was supported by the National Special Research Fund for Non-Profit Sector[grant No. 201303093] and the Shandong Key Research and Development Program, China[grant No. 2018GNC113019]. The funders had no role in study design, data collection, data analysis, data interpretation, the writing of the manuscript or decision to publish.

\section{Availability of data and materials}

The datasets used and analysed during the current study are available from the corresponding author on reasonable request.

Ethics approval and consent to participate

Not applicable.

Consent for publication

Not applicable.

Competing interests

The authors declare that they have no competing interests.

Received: 21 March 2019 Accepted: 24 May 2019

Published online: 30 May 2019

References

1. Barbosa ICR, Hammes UZ, Schwechheimer C. Activation and polarity control of PIN-FORMED auxin transporters by phosphorylation. Trends Plant Sci. 2018;23:523-38.

2. Belteton SA, Sawchuk MG, Donohoe BS, Scarpella E, Szymanski DB. Reassessing the roles of PIN proteins and anticlinal microtubules during pavement cell morphogenesis. Plant Physiol. 2018;176:432-49.

3. Benková E, Michniewicz M, Sauer M, Teichmann T, Seifertová D, Jürgens G, et al. Local, efflux-dependent auxin gradients as a common module for plant organ formation. Cell. 2003;115:591-602. 
4. Cao ZH, Zhang SZ, Wang RK, Zhang RF, Hao YJ. Genome wide analysis of the apple MYB transcription factor family allows the identification of MdoMYB121 gene confering abiotic stress tolerance in plants. PLoS One. 2013:8:e69955.

5. Chen Q, Liu Y, Maere S, Lee E, Van Isterdael G, Xie Z, et al. A coherent transcriptional feed-forward motif model for mediating auxin-sensitive PIN3 expression during lateral root development. Nat Commun. 2015;6:8821.

6. Chen Y, Yang Z, Meng M, Zhao Y, Dong N, Yan H, et al. Cullin mediates degradation of RhoA through evolutionarily conserved BTB adaptors to control actin cytoskeleton structure and cell movement. Mol Cell. 2009;35: 841-55

7. Digby J, Firn RD. The gravitropic set-point angle (GSA): the identification of an important developmentally controlled variable governing plant architecture. Plant Cell Environ. 1995;18:1434-40.

8. Dubos C, Stracke R, Grotewold E, Weisshaar B, Martin C, Lepiniec L. MYB transcription factors in Arabidopsis. Trends Plant Sci. 2010;15:573-81.

9. Evans K, Jung S, Lee T, Brutcher L, Cho I, Peace C, et al. Addition of a breeding database in the genome database for Rosaceae. Database (Oxford). 2013:bat078.

10. Friml J, Wiśniewska J, Benková E, Mendgen K, Palme K. Lateral relocation of auxin efflux regulator PIN3 mediates tropism in Arabidopsis. Nature. 2002; 415:806-9.

11. Hoson T, Soga K. New aspects of gravity responses in plant cells. Int Rev Cytol. 2003;229:209-44

12. Hoson T, Kamisaka S, Masuda Y, Yamashita M. Changes in plant growth processes under microgravity conditions imulated by a three-dimensional clinostat. Bot Mag. 1992;105:53-70.

13. Jung S, Ficklin SP, Lee T, Cheng CH, Blenda A, Zheng P, et al. The genome database for Rosaceae (GDR): year 10 update. Nucleic Acids Res. 2014;42 D1237-44.

14. Kleine-Vehn J, Ding Z, Jones AR, Tasaka M, Morita MT, Friml J. Gravityinduced PIN transcytosis for polarization of auxin fluxes in gravity-sensing root cells. Proc Natl Acad Sci U S A. 2010;107:22344-9.

15. Kumpf RP, Shi CL, Larrieu A, Sto IM, Butenko MA, Peret B, et al. Floral organ abscission peptide IDA and its HAE/HSL2 receptors control cell separation during lateral root emergence. Proc Natl Acad Sci U S A. 2013;110:5235-40

16. Lai LB, Nadeau JA, Lucas J, Lee EK, Nakagawa T, Zhao L, et al. The Arabidopsis R2R3 MYB proteins FOUR LIPS and MYB88 restrict divisions late in the stomatal cell lineage. Plant Cell. 2005;17:2754-67.

17. Lavenus J, Goh T, Roberts I, Guyomarc'h S, Lucas M, De S, et al. Lateral root development in Arabidopsis: fifty shades of auxin. Trends Plant Sci. 2013;18: 450-8.

18. Le J, Liu XG, Yang KZ, Chen XL, Zou JJ, Wang HZ, et al. Auxin transport and activity regulate stomatal patterning and development. Nat Commun. 2014;5: 3090.

19. Livak KJ, Schmittgen TD. Analysis of relative gene expression data using real-time quantitative $P C R$ and the 2(-Delta Delta $C(T))$ method. Methods. 2001;25:402-8.

20. Lucas M, Kenobi K, von Wangenheim D, Vobeta U, Swarup K, De Smet I, et al. Lateral root morphogenesis is dependent on the mechanical properties of the overlaying tissues. Proc Natl Acad Sci U S A. 2013:110:5229-34.

21. Makkena S, Lee E, Sack FD, Lamb RS. The R2R3 MYB transcription factors FOUR LIPS and MYB88 regulate female reproductive development. J Exp Bot. 2012;63:5545-58.

22. Marhavý $P$, Vanstraelen $M$, De Rybel B, Zhaojun D, Bennett MJ, Beeckman T, et al. Auxin reflux between the endodermis and pericycle promotes lateral root initiation. EMBO J. 2013;32:149-58.

23. Martzivanou M, Babbick M, Cogoli-Greuter M, Hampp R. Microgravity-related changes in gene expression after short-term exposure of Arabidopsis thaliana cell cultures. Protoplasma. 2006;229:155-62.

24. Moreno-Risueno MA, Van Norman JM, Moreno A, Zhang J, Ahnert SE, Benfey PN. Oscillating gene expression determines competence for periodic Arabidopsis root branching. Science. 2010;329:1306-11.

25. Pau AL, Zupanska AK, Ostrow DT, Zhang Y, Sun Y, Li JL, et al. Spaceflight transcriptomes: unique responses to a novel environment. Astrobiology. 2012;12:40-56

26. Péret $B$, Li G, Zhao J, Band LR, Voss U, Postaire $O$, et al. Auxin regulates aquaporin function to facilitate lateral root emergence. Nat Cell Biol. 2012; $14: 991-8$.
27. Péret B, Middleton AM, French AP, Larrieu A, Bishopp A, Njo M, et al. Sequential induction of auxin efflux and influx carriers regulates lateral root emergence. Mol Syst Biol. 2013;9:699.

28. Petrásek J, Mravec J, Bouchard R, Blakeslee JJ, Abas M, Seifertová D, et al. PIN proteins perform a rate-limiting function in cellular auxin efflux. Science. 2006;312:914-8.

29. Roychoudhry S, Del Bianco M, Kieffer M, Kepinski S. Auxin controls gravitropic setpoint angle in higher plant lateral branches. Curr Biol. 2013; 23:1497-504.

30. Swarup K, Benkova E, Swarup R, Casimiro I, Peret B, Yang Y, et al. The auxin influx carrier LAX3 promotes lateral root emergence. Nat Cell Biol. 2008;10:946-54.

31. Tamura K, Peterson D, Peterson N, Stecher G, Nei M, Kumar S. MEGA5 molecular evolutionary genetics analysis using maximum likelihood evolutionary distance, and maximum parsimony methods. Mol Biol Evol. 2011:28(10):2731-9.

32. Vanneste S, Coppens F, Lee E, Donner TJ, Xie Z, Van Isterdael G, et al. Developmental regulation of CYCA2s contributes to tissue-specific proliferation in Arabidopsis. EMBO J. 2011;30:3430-41.

33. Vanneste S, Friml J. Auxin: a trigger for change in plant development. Cell. 2009:136:1005-16

34. Vermeer JE, von Wangenheim D, Barberon M, Lee $Y$, Stelzer EH, Maizel A, et al. A spatial accommodation by neighboring cells is required for organ initiation in Arabidopsis. Science. 2014;343:178-83.

35. Vieten A, Vanneste S, Wisniewska J, Benkova E, Benjamins R, Beeckman T, et al. Functional redundancy of PIN proteins is accompanied by auxin-dependent cross-regulation of PIN expression. Development. 2005;132:4521-31.

36. Volkmann D, Baluska F. Gravity: one of the driving forces for evolution. Protoplasma. 2006:229:143-8.

37. Wakabayashi K, Soga K, Hoson T, Kotake T, Kojima M, Sakakibara H, et al Persistence of plant hormone levels in rice shoots grown under microgravity conditions in space: its relationship to maintenance of shoot growth. Physiol Plant. 2017;161:285-93.

38. Wang HZ, Yang KZ, Zou JJ, Zhu LL, Xie ZD, Morita MT, et al. Transcriptional regulation of PIN genes by FOUR LIPS and MYB88 during Arabidopsis root gravitropism. Nat Commun. 2015:6:8822.

39. Wisniewska J, Xu J, Seifertová D, Brewer PB, Ruzicka K, Blilou I, et al. Polar PIN localization directs auxin flow in plants. Science. 2006:312:883.

40. Xie W, Feng Q, Su Y, Dong B, Wu J, Meng L, et al. Transcriptional regulation of PES1 expression by c-Jun in colon cancer. PLoS One. 2012a;7:e42253.

41. Xie XB, Li S, Zhang RF, Zhao J, Chen YC, Zhao Q, et al. The bHLH transcription factor MdbHLH3 promotes anthocyanin accumulation and fruit colouration in response to low temperature in apples. Plant Cell Environ. 2012b;35:1884-97.

42. Xie $Y$, Chen $P$, Yan $Y$, Bao C, Li X, Wang $L$, et al. An atypical R2R3 MYB transcription factor increases cold hardiness by CBF-dependent and CBFindependent pathways in apple. New Phytol. 2017;218:201-18.

43. Xie Z, Lee E, Lucas JR, Morohashi K, Li D, Murray JA, et al. Regulation of cell proliferation in the stomatal lineage by the Arabidopsis MYB FOUR LIPS via direct targeting of core cell cycle genes. Plant Cell. 2010a;22:2306-21.

44. Xie Z, Li D, Wang L, Sack FD, Grotewold E. Role of the stomatal development regulators FLP/MYB88 in abiotic stress responses. Plant J. 2010b;64:731-9.

45. Xuan W, Audenaert D, Parizot B, Moller BK, Njo MF, De Rybel B, et al. Root cap-derived auxin pre-patterns the longitudinal Axis of the Arabidopsis root Curr Biol. 2015;25:1381-8.

46. Yang K, Wang H, Xue S, Qu X, Zou J, Le J. Requirement for A-type cyclindependent kinase and cyclins for the terminal division in the stomatal lineage of Arabidopsis. J Exp Bot. 2014:65:2449-61.

47. Zhang W, Cao Z, Zhou Q, Chen J, Xu G, Gu J, et al. Grain filling characteristics and their relations with endogenous hormones in large- and small-grain mutants of Rice. PLoS One. 2016;11:e0165321.

48. Zhou JJ, Luo J. The PIN-FORMED auxin efflux carriers in plants. Int J Mol Sci. 2018;19.

49. Zhou LJ, Zhang CL, Zhang RF, Wang GL, Li YY, Hao YJ. The SUMO E3 ligase MdSIZ1 targets MdbHLH104 to regulate plasma membrane H(+)-ATPase activity and Iron homeostasis. Plant Physiol. 2019;179:88-106.

\section{Publisher's Note}

Springer Nature remains neutral with regard to jurisdictional claims in published maps and institutional affiliations. 